to revisiting information transfer between neurons, with the proposed concept of a "tripartite synapse" (Perea and Araque, 2002).

In this issue, MacDonald et al. (2008) focus on the astrocyte-to-astrocyte communication, by exploring the long-range propagation mechanisms of intracellular calcium waves (ICW). These represent a form of intercellular signaling in astrocyte networks, where ATP release is initiated by the intracellular $\mathrm{Ca}^{2+}$ elevation, and whose diffusion acts as cell-to-cell transmitter. Authors employ in vitro calcium imaging in astrocytes cultures and a simple phenomenological mathematical model. Through such a combined approach, they examine two apparently conflicting scenarios, as debated in the literature: during ICW, (1) ATP is secreted by one cell (i.e., a point-source) and diffuses activating nearby astrocytes, or (2) ATP-induced cell activation is actively regenerated by downstream astrocytes, which in turn secrete ATP, similarly to propagation of an action-potential through a myelinated axon.

Only through a combination of (1) and (2), resulting in both regenerative and diffusive mechanisms, the model is able to match quantitatively the in vitro ICW. This result reconciles elegantly the previous debate between single-point source models and fully regenerative signaling models, and it is particularly significant due to the extremely simple nature of the model. Even though several biophysical details underlying ATP diffusion and single-cell "excitability" were neglected, this model allows for systematic interpretation and analysis of the relationship between single-cell and network emerging properties, which is hard to obtain in accurate biophysical descriptions.

It is intriguing to outline the similarity between the astrocyte model introduced here, and the spiking network models of the integrate-and-fire family. The last are reduced models of neuronal excitability, shown to capture quantitatively the experimental responses from cells in large networks (Jolivet et al., 2008). Similarly, MacDonald et al. (2008) identifies a minimal description to predict network-level ICW propagation. These considerations therefore suggest that the two modeling approaches may be unified, incorporating spiking neurons, chemical synapses as well as astrocytes, ICW and ATP-diffusion. The results of such attempts might be valuable to identify global activity regimes, to be explored in greater details through large-scale simulations (Markram, 2006) that incorporate anatomically precise details on neurons, astrocytes and the intercellular environment (Helmstaedter et al., 2009).

\section{REFERENCES}

Fellin, T. (2009). Communication between neurons and astrocytes: relevance to the modulation of synaptic and network activity. J. Neurochem. 108, 533-544.

Helmstaedter, M., Briggman, K. L., and Denk, W. (2009). 3D structural imaging of the brain with photons and electrons. Curr. Opin. Neurobiol. 18, 633-641.

Jolivet, R., Schürmann, F., Berger, T. K., Naud, R., Gerstner, W., and Roth, A. (2008). The quantitative single-neuron modeling competition. Biol. Cybern. $99,417-426$.

MacDonald, C. L., Yu, D., Buibas, M., and Silva, G. A. (2008). Diffusion modeling of ATP signaling suggests a partially regenerative mechanism underlies astrocyte intercellular calcium waves. Front. Neuroeng. doi: 10.3389/neuro.16.001.2008.

Markram, H. (2006). The blue brain project. Nat. Rev. Neurosci. 7, 153-160.

Ni, Y., Malarkey, E. B., and Parpura, V. (2007). Vesicular release of glutamate mediates bidirectional signaling between astrocytes and neurons. J. Neurochem. 103, 1273-1284.

Perea, G., and Araque, A. (2002). Communication between astrocytes and neurons: a complex language. J. Physiol Paris 96, 199-207.

Received: 04 May 2009; published: 15 September 2009. Citation: Front. Neurosci. (2009) 3, 2: 160-161. doi: 10.3389/neuro.01.019.2009

Copyright $\odot 2009$ Giugliano. This is an open-access publication subject to an exclusive license agreement between the authors and the Frontiers Research Foundation, which permits unrestricted use, distribution, and reproduction in any medium, provided the original authors and source are credited.

\title{
Genes and cognitive aging
}

\section{William Jagust*}

Helen Wills Neuroscience Institute, University of California, Berkeley, Berkeley, CA, USA

*Correspondence: jagust@berkeley.edu

\section{A commentary on}

Genetic contributions to age-related decline in executive function: a 10 -year longitudinal study of COMT and BDNF polymorphisms

by Kirk I. Erickson, Jennifer S. Kim, Barbara L. Suever, Michelle W. Voss, B. Magnus Francis and Arthur F. Kramer

The search for genetic factors related to late life cognitive change initially focused on cognitive diseases, particularly Alzheimer's disease (AD) and now increasingly employs the method of genome-wide association studies. This approach has repeatedly confirmed the major importance of the Apolipoprotein E polymorphism as a risk factor for $\mathrm{AD}$, but no consensus has emerged concerning other genes. The reasons for this situation likely include factors such as the large number of potential candidate genes with small effects, interactions between genes, and the difficulty of establishing a reli- able and valid phenotypic marker of both disease and normality.

The field of cognitive aging is also grappling with the genetic determinants of cognitive change, using different approaches in the face of a unique set of challenges. Because cognitive aging is probably even more heterogeneous than a cognitive disorder like $\mathrm{AD}$, definition of the precise phenotype is problematic. Factors that can define this phenotype include the exact cognitive process and a host of demographic factors. In addition, the small effect sizes and poten- 
tial secular or cohort effects would seem to necessitate a longitudinal approach.

Rising to these challenges, Erickson et al. (2008) have produced an interesting set of findings that examined polymorphic alleles in the catechol-O-methyl transferase (COMT) gene and the brain-derived neurotrophic factor (BDNF) gene. Both genes have functional single nucleotide polymorphisms. In the case of COMT a valine to methionine substitution at codon 158 results in a met/met form with low thermostability, less enzymatic degradation of prefrontal dopamine (DA) and thus higher prefrontal DA function than the val/val form. While many studies of both young and old individuals find that met homozygotes perform better on tests of prefrontal function than val homozygotes, these results are not consistent. BDNF is characterized by a met/val substitution at codon 66 in which the met form of the allele results in impaired BDNF trafficking that appears to coincide with reductions in both cognitive performance and brain volumes in young people although, again, results disagree. Erickson and colleagues used a longitudinal, withinsubjects design to evaluate how these polymorphisms affected change in performance on a task-switching paradigm in a group of 53 individuals studied at baseline at an average age of 65 and then again 10 years later. In short, the authors found no effect of the COMT polymorphism on the task, but they did find an effect of the BDNF polymorphism. Interestingly, the effect, revealed as a time $\times$ genotype interaction, such that those homozygous for the val allele had greater decline in performance than those with a met allele.

In the same issue of this journal, Nagel et al. (2008) used a cross-sectional approach and looked at two different cognitive tasks (the Wisconsin Card Sorting Test and a spatial working memory task) and found strong evidence of both an age $\times$ COMT genotype (such that older subjects with the Val allele were poorer performers than Met homozygotes) and a three-way interaction with the BDNF allele in which the Met allele in COMT Val carriers was detrimental.

These results follow on previous studies that have been inconsistent in many ways. Neither of these studies noted effects of the COMT genotype on performance in young people, despite the fact that a benefit of the Met allele has been frequently seen on a variety of frontal and executive tasks including the WCST, as well as on brain activation during such tasks in both young and old (Egan et al., 2001; Goldberg and Weinberger, 2004; Malhotra et al., 2002; Starr et al., 2007). However, COMT genotype has not invariably shown this effect in either young or older individuals, and effects are often small, vary by gender or the precise cognitive task, and may differ between Met homozygotes and heterozygotes (Barnett et al., 2007; Harris et al., 2005; Holtzer et al., 2008; O'Hara et al., 2006). A key question is whether longitudinal studies of older people show different results. Reports have noted beneficial effects of the Met/Met genotype (Starr et al., 2007) and greater declines in those with $\mathrm{Val} / \mathrm{Val}$ (de Frias et al., 2004, 2005). However, these effects also vary according to the specific tests, and the specific age of the subjects. With regard to BDNF, some studies of older people show better cognitive performance in Met homozygotes (Harris et al., 2006), and others worse (Raz et al., 2009).

Why are results of these studies so inconsistent? One possibility is that these genes have relatively small effects so that the precise specification of the phenotype is crucial. For example, the cognitive tasks assessed in these studies have involved either tests of visuospatial ability, executive function (and very different measures of this broad category), episodic memory, reasoning ability, or speed of processing. It does not necessarily make any more sense to compare genetic influences on these processes to one another than it does to compare the genetics of Alzheimer's and Parkinson's disease. In addition, findings differ by gender and age, and age itself is a complicated variable that may be measured cross-sectionally, longitudinally, and at many different time points and ranges. Furthermore, evidence suggests that the polymorphisms not only interact with basic demographic features, but with comorbid disease such that, for example, individuals with high blood glucose show poorer memory function only if they carry the Met BDNF allele (Raz et al., 2008). And, of course there are gene-gene interactions. These issues are not limited to genes with small effects, as the Apolipoprotein E4 allele, repeatedly demonstrated as a risk for cognitive decline and dementia in older people, may be beneficial in younger individuals (Mondadori et al., 2007).
The precise specification of a demographic and behavioral phenotype in association with genetic variations is clearly one way the field is moving forwards. Another way is through the use of other phenotypic markers such as brain imaging (Pezawas et al., 2004) that may be a reliable endophenotype. Here again, if applied carefully, associations between changes in brain structure and function may help to explain not only genetic associations, but behavioral mechanisms. It seems reasonable that only by precisely specifying a cognitive or imaging phenotype and performing studies in well characterized individuals will the genetics of cognitive aging advance.

\section{REFERENCES}

Barnett, J. H., Jones, P. B., Robbins, T. W., and Muller, U. (2007). Effects of the catechol-O-methyltransferase Val158Met polymorphism on executive function: a meta-analysis of the Wisconsin Card Sort Test in schizophrenia and healthy controls. Mol. Psychiatry 12, 502-509.

de Frias, C. M., Annerbrink, K., Westberg, L., Eriksson, E., Adolfsson, R., and Nilsson, L. G. (2004). COMT gene polymorphism is associated with declarative memory in adulthood and old age. Behav. Genet. 34, 533-539.

de Frias, C. M., Annerbrink, K., Westberg, L., Eriksson, E., Adolfsson, R., and Nilsson, L. G. (2005). Catechol $\mathrm{O}$-methyltransferase Val158Met polymorphism is associated with cognitive performance in nondemented adults. J. Cogn. Neurosci. 17, 1018-1025.

Egan, M. F., Goldberg, T. E., Kolachana, B. S., Callicott, J. H., Mazzanti, C. M., Straub, R. E., Goldman, D., and Weinberger, D. R. (2001). Effect of COMT Val108/158 Met genotype on frontal lobe function and risk for schizophrenia. Proc. Natl. Acad. Sci. USA 98, 6917-6922.

Erickson, K. I., Kim, J. S., Suever, B. L., Voss, M. W., Francis, B. M., and Kramer, A. F. (2008). Genetic contributions to age-related decline in executive function: a 10-year longitudinal study of COMT and BDNF polymorphisms. Front. Hum. Neurosci. 2, 11.

Goldberg, T. E., and Weinberger, D. R. (2004). Genes and the parsing of cognitive processes. Trends Cogn. Sci. 8, 325-335.

Harris, S. E., Fox, H., Wright, A. F., Hayward, C., Starr, J. M., Whalley, L. J., and Deary, I. J. (2006). The brain-derived neurotrophic factor Val66Met polymorphism is associated with age-related change in reasoning skills. Mol. Psychiatry 11, 505-513.

Harris, S. E., Wright, A. F., Hayward, C., Starr, J. M., Whalley, L. J., and Deary, I. J. (2005). The functional COMT polymorphism, Val $158 \mathrm{Met}$, is associated with logical memory and the personality trait intellect/imagination in a cohort of healthy 79 year olds. Neurosci. Lett. 385, 1-6.

Holtzer, R., Ozelius, L., Xue, X., Wang, T., Lipton, R. B., and Verghese, J. (2008). Differential effects of COMT on gait and executive control in aging. Neurobiol. Aging. doi: 10.1016/jneurobiolaging.2008.05.011. [Epub ahead of print]. 
Malhotra, A. K., Kestler, L. J., Mazzanti, C., Bates, J. A., Goldberg, T., and Goldman, D. (2002). A functional polymorphism in the COMT gene and performance on a test of prefrontal cognition. Am. J. Psychiatry $159,652-654$.

Mondadori, C. R., de Quervain, D. J., Buchmann, A., Mustovic, H., Wollmer, M. A., Schmidt, C. F., Boesiger, P., Hock, C., Nitsch, R.M., Papassotiropoulos, A., and Henke, K. (2007). Better memory and neural efficiency in young apolipoprotein E epsilon 4 carriers. Cereb. Cortex 17, 1934-1947.

Nagel, I. E., Chicherio, C., Li, S. C., von Oertzen, T., Sander, T., Villringer, A., Heekeren, H. R., Backman, L., and Lindenberger, U. (2008). Human aging magnifies genetic effects on executive functioning and working memory. Front. Hum. Neurosci. 2, 1.
O'Hara, R., Miller, E., Liao, C. P., Way, N., Lin, X., and Hallmayer, J. (2006). COMT genotype, gender and cognition in community-dwelling, older adults. Neurosci. Lett. 409, 205-209.

Pezawas, L., Verchinski, B. A., Mattay, V. S., Callicott, J. H., Kolachana, B. S., Straub, R. E., Egan, M. F., MeyerLindenberg, A., and Weinberger, D. R. (2004). The brain-derived neurotrophic factor val66met polymorphism and variation in human cortical morphology. J. Neurosci. 24, 10099-10102.

Raz, N., Dahle, C. L., Rodrigue, K. M., Kennedy, K. M., Land, S. J., and Jacobs, B. S. (2008). Brain-derived neurotrophic factor Val66Met and blood glucose: a synergistic effect on memory. Front. Hum. Neurosci. 2, 12.

Raz, N., Rodrigue, K. M., Kennedy, K. M., and Land, S. (2009). Genetic and vascular modifiers of age-sensi- tive cognitive skills: effects of COMT, BDNF, ApoE, and hypertension. Neuropsychology 23, 105-116.

Starr, J. M., Fox, H., Harris, S. E., Deary, I. J., and Whalley, L. J. (2007). COMT genotype and cognitive ability: a longitudinal aging study. Neurosci. Lett. 421, 57-61.

Received: 02 May 2009; published: 15 September 2009. Citation: Front. Neurosci. (2009) 3, 2: 161-163. doi: 10.3389/neuro.01.020.2009

Copyright (C) 2009 Jagust. This is an open-access publication subject to an exclusive license agreement between the authors and the Frontiers Research Foundation, which permits unrestricted use, distribution, and reproduction in any medium, provided the original authors and source are credited.

\section{A primer of visual stimulus presentation software}

\section{Rolf Kötter*}

Section Neurophysiology and Neuroinformatics (126), Centre for Neuroscience, Donders Institute for Brain, Cognition, and Behaviour, Radboud University Nijmegen Medical Centre, Nijmegen, The Netherlands *Correspondence: rk@donders.ru.nl

\section{A commentary on}

Vision Egg: an open-source library for realtime visual stimulus generation

by Andrew D. Straw

The visual system has been the most widely studied sensory system in neuroscience during the last decades. A reliable and flexible visual stimulus presentation tool is one of the most important prerequisites for a thorough analysis of its sensory processing characteristics. While almost all sensory systems labs have created some home-grown solutions, these are not easily transferable from one lab to another or from one presentation platform to another. In addition, several stimuli are hard to generate with the desired accuracy in timing, color and luminance, $3 \mathrm{D}$ rendering or stereopsis.

Vision Egg (Straw, 2008) is a more widely used software library, designed originally to probe the visual system of the fly. It is an open source and platform-independent software package built on top of Python (as the programming language) and OpenGL (for graphics instructions). For a well versed programmer, Vision Egg achieves its goals very well, providing a powerful and highly optimized system for visual stimulus presentation and interactions with hardware -

\begin{tabular}{rrrr} 
Table 1 | Comparison of several frequently used software packages for visual stimulus presentation. \\
\hline Vision Egg $\quad$ PsychoPy & Psychtoolbox & Presentation
\end{tabular}

\begin{tabular}{lcccc} 
Full source code & $\checkmark \checkmark$ & $\checkmark \checkmark$ & $\checkmark$ (None for Matlab) & - \\
\hline Platform independent & $\checkmark \checkmark$ & $\checkmark \checkmark$ & $\checkmark \checkmark$ & - \\
Usability & $\checkmark$ & $\checkmark \checkmark$ & $\checkmark \checkmark$ & - \\
\hline Automated calibration & - & $\checkmark \checkmark \checkmark$ & $\checkmark$ & - \\
Stimuli in realtime & $\checkmark \checkmark$ & $\checkmark \checkmark$ & $\checkmark \checkmark$ & $\checkmark \checkmark$ \\
Hardware interfaces & $\checkmark \checkmark$ & $\checkmark \checkmark$ & $\checkmark \checkmark$ & $\checkmark \checkmark$ \\
Community size & $\checkmark \checkmark$ & $\checkmark \checkmark$ & $\checkmark$ (Requires Matlab) & - \\
Free & $\checkmark \checkmark$ (Python, OpenGL) & $\checkmark$ (Python) & &
\end{tabular}

including the ability to run experiments remotely across a network (via TCP/IP). While historically the Vision Egg software strongly adheres to an object-oriented model of programming this can be hard to understand for relatively inexperienced programmers. For instance, the temporal control of experiments in Vision Egg is predominantly through the use of presentation loops, whereby the user sets an object to run for a given length of time, attaches stimuli to it, assigns it to a screen, and then tells the object to "go". This "mainloop-andcallback" mechanism of flow control has advantages where stimuli continue to run between trials. The alternative, however, of an explicit sequence of control statements can also be implemented (see Figure 2 of Straw, 2008).

Table 1 (adapted from Peirce, 2007) gives a comparison of various features of four well known stimulus presentation programs. Two of these (Vision Egg and PsychoPy) have very similar philosophies, are both implemented in Python, and differed originally in their low-latency realtime capabilities. The most substantive differences between them today are that Vision Egg offers relatively simple perspective corrected stimuli utilizing the $3 \mathrm{D}$ 